\title{
Bounds on canonical Green's functions
}

\author{
J. Jorgenson and J. Kramer
}

\begin{abstract}
A fundamental object in the theory of arithmetic surfaces is the Green's function associated to the canonical metric. Previous expressions for the canonical Green's function have relied on general functional analysis or, when using specific properties of the canonical metric, the classical Riemann theta function. In this article, we derive a new identity for the canonical Green's function involving the hyperbolic heat kernel. As an application of our results, we obtain bounds for the canonical Green's function through covers and for families of modular curves.
\end{abstract}

\section{Introduction}

\section{1}

In [Ara74], Arakelov defined an intersection theory for divisors on arithmetic surfaces by including a contribution at infinity, which is computed using certain Green's functions defined on the corresponding Riemann surfaces. Arakelov's theory has been extended to higher dimensions, primarily through the work of H. Gillet, C. Soulé, and G. Faltings. Motivated by the recent work of B. Edixhoven, which will be explained below, we derive here several analytic relations and estimates for the Green's functions used by Arakelov.

More specifically, let $X$ be a compact Riemann surface of genus $g_{X}>1$. The canonical volume form $\mu_{\text {can }}$ on $X$ is the positive $(1,1)$-form obtained by the pull-back of the standard Euclidean form on the Jacobian variety $\operatorname{Jac}(X)$ associated to $X$ via the classical Abel-Jacobi map. The canonical Green's function $g_{\mathrm{can}}(z, w)$, also written as $g_{\mathrm{can}, X}(z, w)$, is the function of $z, w \in X$, which is uniquely characterized by the differential equation

$$
d_{z} d_{z}^{c} g_{\mathrm{can}}(z, w)+\delta_{w}(z)=\mu_{\mathrm{can}}(z) \quad(z, w \in X),
$$

where $\delta_{w}(z)$ is the usual Dirac delta distribution, and the normalization condition

$$
\int_{X} g_{\mathrm{can}}(z, w) \mu_{\mathrm{can}}(z)=0 \quad(w \in X) .
$$

The fundamental properties of the canonical Green's function, such as existence and symmetry, follow from general functional analysis. By identifying the points $z, w \in X$ with their pre-images in the universal cover, which we take to be the hyperbolic upper half-plane $\mathbb{H}$, we have that the function

$$
g_{\text {can }}(z, w)+\log |z-w|^{2}
$$

is bounded and continuous as $z$ approaches $w$.

Received 25 April 2005, accepted in final form 16 September 2005.

2000 Mathematics Subject Classification 14G40, 30F10, 11F72, 30C40.

Keywords: Green's functions, Arakelov theory, modular curves, hyperbolic heat kernels.

The first author acknowledges support from NSF and PSC-CUNY grants.

This journal is (C) Foundation Compositio Mathematica 2006. 


\section{J. Jorgenson And J. Kramer}

The results we present here involve a development of bounds for the canonical Green's function after removing its logarithmic singularity. In effect, we obtain three types of bound. First, we study the setting of a fixed compact hyperbolic Riemann surface $X$, ultimately deriving a sup-norm bound involving quantities associated to the hyperbolic spectral theory and hyperbolic geometry on $X$. Second, we investigate these bounds in the relative situation, when $X$ is a finite-degree cover of a fixed compact hyperbolic Riemann surface. Third, we consider these bounds for families of hyperbolic modular curves, meaning the sequences of modular curves $\left\{X_{0}(N)\right\},\left\{X_{1}(N)\right\}$, or $\{X(N)\}$ of genus bigger than one.

To prove our results, we develop the bounds by first deriving bounds for the difference between the canonical Green's function and the hyperbolic Green's function, whose definition parallels that of the canonical Green's function when replacing the canonical $(1,1)$-form by the appropriately scaled hyperbolic $(1,1)$-form. We then express the difference between the canonical and the hyperbolic Green's functions using various expressions involving the hyperbolic heat kernel (including special values of Selberg's zeta function). The remainder of the article is devoted to proving bounds for hyperbolic heat kernels, from which our main results follow.

\subsection{Arithmetic applications}

Analytic problems related to Arakelov theory can be interesting both for their own sake and for potential applications to arithmetic algebraic geometry. Concerning the specific work we undertake in the present article, we were informed of some analytic problems with immediate arithmetic implications in current work by Edixhoven, which we now briefly discuss.

Edixhoven has a strategy to compute Galois representations modulo $\ell$ associated to a fixed modular form of arbitrary weight, with the goal of devising an algorithm, which has complexity that is polynomial in $\ell$. A typical modular form to consider is $\Delta$, the (up to scale) unique cusp form of weight 12 associated to the modular group $\mathrm{PSL}_{2}(\mathbb{Z})$. In this case, Edixhoven's strategy amounts to computing the field of definition of a suitable torsion point of order $\ell$ on the Jacobian variety $\operatorname{Jac}\left(X_{1}(\ell)\right)$ of the modular curve $X_{1}(\ell)$. Naturally, such torsion points can be described in terms of a divisor on $X_{1}(\ell)$. Since the dimension of $\operatorname{Jac}\left(X_{1}(\ell)\right)$ grows quadratically with $\ell$, it seems as if existing methods to compute torsion points, such as with computer algebra systems, will be unfeasible. Edixhoven's idea is to numerically approximate the divisor in question with sufficiently high precision so that the approximation can turn into an exact result. More precisely, in order to get a polynomial time algorithm, one needs that the precision in the above approximation (that is, the number of digits with which the numerical computations need to be carried out) is to be at most polynomial in $\ell$.

In Edixhoven's work, the required precision is roughly equal to the height of the divisor, which is estimated using Arakelov theory. The arithmetic Riemann-Roch theorem, Noether's formula, and estimates for the Faltings height of $X_{1}(\ell)$ and for norms of theta functions are applied. To complete this analysis, Edixhoven needs various estimates involving an upper bound for Green's functions on $X_{1}(\ell)$, as a function of $\ell$. As an application of our general results, we derive an upper bound for the Green's functions on $X_{1}(\ell)$, after removing its logarithmic singularity. Indeed, our upper bound is uniform in $\ell$, thus showing that the analytic contribution from the Green's functions in Edixhoven's algorithm is an order smaller than required by the algorithm.

In communicating his ideas, Edixhoven informed us that F. Merkl has studied methods, which yield upper bounds for Green's functions, that are polynomial in $\ell$. Our method of proof, which builds on previous investigations, notably [JK01, JK04a, JK04b], provides a sharper upper bound, which we hope will lead to a better estimate of the complexity of Edixhoven's algorithm. 


\section{BOUNDS ON CANONICAL GREEN'S FUNCTIONS}

\subsection{Summary of the main results}

The hyperbolic Green's function $g_{\text {hyp }}(z, w)$ on $X$ is the function of $z, w \in X$, which satisfies the differential equation

$$
d_{z} d_{z}^{c} g_{\mathrm{hyp}}(z, w)+\delta_{w}(z)=\frac{\mu_{\mathrm{hyp}}(z)}{\operatorname{vol}_{\mathrm{hyp}}(X)} \quad(z, w \in X),
$$

and the normalization condition

$$
\int_{X} g_{\mathrm{hyp}}(z, w) \mu_{\mathrm{hyp}}(z)=0 \quad(w \in X),
$$

where $\mu_{\text {hyp }}$ is the $(1,1)$-form associated to the metric with constant negative curvature equal to minus one giving $X$ the volume $\operatorname{vol}_{\text {hyp }}(X)$. In particular, if $z, w \in \mathbb{H}$, the hyperbolic Green's function on $\mathbb{H}$ is given by

$$
g_{\mathbb{H}}(z, w)=-\log \left(\left|\frac{z-w}{z-\bar{w}}\right|^{2}\right) .
$$

Our first main result, Theorem 3.8, expresses the difference $g_{\mathrm{can}}(z, w)-g_{\mathrm{hyp}}(z, w)$ in terms of a function associated to hyperbolic geometry, namely the hyperbolic heat kernel on $X$. This construction of $g_{\text {can }}(z, w)$ allows for the study of the canonical Green's function through techniques of hyperbolic geometry. We then study the identity from Theorem 3.8 and prove bounds for the hyperbolic Green's function and the canonical Green's function on $X$ in terms of small eigenvalues and corresponding eigenfunctions of the hyperbolic Laplacian on $X$, as well as other data coming from hyperbolic geometry, such as the length of the shortest closed geodesic and the injectivity radius of $X$. These results are summarized in Theorems 4.5, 4.8, and 4.9.

We then study these bounds for families of compact hyperbolic Riemann surfaces. In general, let $X_{1}$ be a finite degree cover of $X_{0}$, a fixed compact hyperbolic Riemann surface. Let $g_{X_{1}}$ denote the genus of $X_{1}$ and $\lambda_{X_{1}, 1}$ be the smallest non-zero eigenvalue of the hyperbolic Laplacian on $X_{1}$. Given a uniformization $X_{1}=\Gamma_{X_{1}} \backslash \mathbb{H}$ (with $\Gamma_{X_{1}}$ a cocompact torsion-free Fuchsian subgroup of the first kind of $\mathrm{PSL}_{2}(\mathbb{R})$ ), we shall, by abuse of notation, identify $X_{1}$ with a choice of a fundamental domain for $X_{1}$ in $\mathbb{H}$, and identify points on $X_{1}$ with their pre-images in $\mathbb{H}$. Given $\delta>0$, and points $z, w \in X_{1}$, define the set

$$
S_{\Gamma_{X_{1}}}(\delta ; z, w)=\left\{\gamma \in \Gamma_{X_{1}} \mid d_{\mathbb{H}}(z, \gamma w)<\delta\right\} ;
$$

here $d_{\mathbb{H}}(\cdot, \cdot)$ denotes the hyperbolic distance on $\mathbb{H}$. Let $\left\{\lambda_{X_{1}, n}\right\}$ denote the set of eigenvalues of the hyperbolic Laplacian, which acts on the space of smooth functions on $X_{1}$, with associated orthonormal eigenfunctions $\left\{\varphi_{X_{1}, n}\right\}$. We prove that for any $\varepsilon>0, \delta>0$, and for all $z, w \in X_{1}$, we have the bounds

$$
g_{\mathrm{hyp}, X_{1}}(z, w)-\sum_{\gamma \in S_{\Gamma_{X_{1}}}(\delta ; z, w)} g_{\mathbb{H}}(z, \gamma w)-\sum_{0<\lambda_{X_{1}, n} \leqslant \varepsilon} \frac{4 \pi}{\lambda_{X_{1}, n}} \varphi_{X_{1}, n}(z) \varphi_{X_{1}, n}(w)=O_{X_{0}, \varepsilon, \delta}(1),
$$

and

$$
g_{\text {can }, X_{1}}(z, w)-g_{\mathrm{hyp}, X_{1}}(z, w)=O_{X_{0}}\left(\frac{1}{g_{X_{1}}}\left(1+\frac{1}{\lambda_{X_{1}, 1}}\right)\right)
$$

therefore, by the triangle inequality, we show that

$$
g_{\mathrm{can}, X_{1}}(z, w)-\sum_{\gamma \in S_{\Gamma_{X_{1}}}(\delta ; z, w)} g_{\mathbb{H}}(z, \gamma w)=O_{X_{0}, \delta}\left(1+\frac{1}{\lambda_{X_{1}, 1}}\right) .
$$

As the notation indicates, all bounds are uniform on $X_{1}$, and depend solely on the choices of $\varepsilon, \delta$, and the base surface $X_{0}$. The proofs of these bounds are given in $\S 5$. 


\section{J. Jorgenson And J. KRAmer}

As in [JK04b], we extend our analysis to the study of the families of hyperbolic modular curves $\left\{X_{0}(N)\right\},\left\{X_{1}(N)\right\}$, and $\{X(N)\}$. In this setting, it was shown in [Bro99] that the smallest non-zero eigenvalues are uniformly bounded away from zero. Therefore, our results imply, among others, the estimates

$$
g_{\mathrm{can}, X_{1}(N)}(z, w)-g_{\mathrm{hyp}, X_{1}(N)}(z, w)=O\left(g_{X_{1}(N)}^{-1}\right),
$$

and

$$
g_{\text {can }, X_{1}(N)}(z, w)-\sum_{\gamma \in S_{\Gamma_{X_{1}(N)}}(\delta ; z, w)} g_{\mathbb{H}}(z, \gamma w)=O_{\delta}(1),
$$

with similar bounds for the other families of modular curves $\left\{X_{0}(N)\right\}$ and $\{X(N)\}$. Again, as the notation indicates, the bounds are uniform in $N$.

\subsection{Outline of the paper}

The article is organized as follows. In $\S 2$, we establish our notation and discuss background material and results. In $\S 3$, we derive an explicit, analytic expression relating the canonical Green's function to the hyperbolic Green's function and various other data coming from hyperbolic geometry. For the most part, the data from hyperbolic geometry that we use come directly from integral expressions involving the hyperbolic heat kernel, including the special value of the Selberg zeta function, which was studied in [JK02]. The main formula we derive is stated in Theorem 3.8. In $\S 4$, we bound all quantities appearing in Theorem 3.8 in terms of fundamental invariants from hyperbolic geometry, such as the smallest non-zero eigenvalue, the length of the shortest closed geodesic, etc.; a list summarizing the invariants, which we use, is given in $\S 2.6$. In $\S 5$, we study the behavior of these invariants in two different settings, namely, a compact Riemann surface $X_{1}$, which is a finite degree cover of some fixed compact hyperbolic Riemann surface $X_{0}$, or a compact Riemann surface $X_{1}$, which lies in one of the families of hyperbolic modular surfaces $\left\{X_{0}(N)\right\},\left\{X_{1}(N)\right\}$, or $\{X(N)\}$. The analysis of many of the hyperbolic invariants that appear in the present article have also been studied in detail in [JK04b]. The corresponding results of [JK04b] are then applied to the bounds obtained in $\S 4$, thus completing the proofs of the results stated above.

\section{Background material}

\subsection{Hyperbolic and canonical metrics}

Let $\Gamma$ be a Fuchsian subgroup of the first kind of $\mathrm{PSL}_{2}(\mathbb{R})$ acting by fractional linear transformations on the hyperbolic upper half-plane, which we denote by $\mathbb{H}=\{z \in \mathbb{C} \mid \operatorname{Im}(z)>0\}$. We let $X$ be the quotient space $\Gamma \backslash \mathbb{H}$ and denote by $g_{X}$ the genus of $X$. In a slight abuse of notation, throughout this article we identify $X$ with a fundamental domain (say, a Ford domain, bounded by geodesic paths) and identify points on $X$ with their pre-images in $\mathbb{H}$. We assume that $g_{X}>1$ and that $\Gamma$ has no elliptic and, apart from the identity, no parabolic elements, that is, $X$ is smooth and compact.

In the following, $\mu$ denotes a (smooth) metric on $X$, that is, $\mu$ is a positive $(1,1)$-form on $X$. We write $\operatorname{vol}_{\mu}(X)$ for the volume of $X$ with respect to $\mu$. In particular, we let $\mu=\mu_{\text {hyp }}$ denote the hyperbolic metric on $X$, which is compatible with the complex structure of $X$, and has constant negative curvature equal to minus one. Locally, we have

$$
\mu_{\mathrm{hyp}}(z)=\frac{i}{2} \cdot \frac{d z \wedge d \bar{z}}{\operatorname{Im}(z)^{2}}
$$

As a shorthand, we write $v_{X}$ for the hyperbolic volume $\operatorname{vol}_{\mu_{\text {hyp }}}(X)$; we recall that $v_{X}$ is given by $4 \pi\left(g_{X}-1\right)$. The scaled hyperbolic metric $\mu=\mu_{\text {shyp }}$ is simply the rescaled hyperbolic metric $\mu_{\text {hyp }} / v_{X}$, which measures the volume of $X$ to be one. 


\section{BOUNDS ON CANONICAL GREEN'S FUNCTIONS}

Let $S_{k}(\Gamma)$ denote the $\mathbb{C}$-vector space of cusp forms of weight $k$ with respect to $\Gamma$ equipped with the Petersson inner product

$$
\langle f, g\rangle=\frac{i}{2} \int_{X} f(z) \overline{g(z)} \operatorname{Im}(z)^{k} \cdot \frac{d z \wedge d \bar{z}}{\operatorname{Im}(z)^{2}} \quad\left(f, g \in S_{k}(\Gamma)\right) .
$$

By choosing an orthonormal basis $\left\{f_{1}, \ldots, f_{g_{X}}\right\}$ of $S_{2}(\Gamma)$ with respect to the Petersson inner product, the canonical metric $\mu=\mu_{\text {can }}$ of $X$ is given by

$$
\mu_{\text {can }}(z)=\frac{1}{g_{X}} \cdot \frac{i}{2} \sum_{j=1}^{g_{X}}\left|f_{j}(z)\right|^{2} d z \wedge d \bar{z} .
$$

We note that the canonical metric measures the volume of $X$ to be one. For the purpose of comparing the hyperbolic and the canonical metrics, we define

$$
d_{X}=\sup _{z \in X}\left|\frac{\mu_{\text {can }}(z)}{\mu_{\text {shyp }}(z)}\right| .
$$

In [JK04a], optimal bounds for $d_{X}$ through covers were obtained for arbitrary towers of compact and non-compact Riemann surfaces; see also [Don96], where the author considered the problem of towers of compact Riemann surfaces.

\subsection{Green's functions and residual metrics}

We denote the Green's function associated to the metric $\mu$ by $g_{\mu}$. It is a function on $X \times X$ characterized by the two properties

$$
\begin{gathered}
d_{z} d_{z}^{c} g_{\mu}(z, w)+\delta_{w}(z)=\frac{\mu(z)}{\operatorname{vol}_{\mu}(X)} \\
\int_{X} g_{\mu}(z, w) \mu(z)=0 \quad(w \in X) .
\end{gathered}
$$

Assuming that $z, w$ are points on $X$, which are sufficiently close, our convention for the Green's function is such that the sum $g_{\mu}(z, w)+\log |z-w|^{2}$ is bounded as $w$ approaches $z$.

The Green's function is an integral kernel that inverts the Laplacian associated to $\mu$ and is orthogonal to the constant functions. More precisely, for any smooth, bounded function $f$ on $X$, we have the identity

$$
\int_{X} g_{\mu}(z, \zeta)\left(-d_{\zeta} d_{\zeta}^{c} f(\zeta)\right) \mu(\zeta)=f(z), \text { provided that } \int_{X} f(\zeta) \mu(\zeta)=0
$$

If $\mu=\mu_{\text {hyp }}, \mu=\mu_{\text {shyp }}$, or $\mu=\mu_{\text {can }}$, we set

$$
g_{\mu}=g_{\mathrm{hyp}}, \quad g_{\mu}=g_{\mathrm{shyp}}, \quad g_{\mu}=g_{\mathrm{can}},
$$

respectively. By means of the function $G_{\mu}=\exp \left(g_{\mu}\right)$, we can now define a metric $\|\cdot\|_{\mu \text {,res }}$ on the canonical line bundle $\Omega_{X}^{1}$ of $X$ in the following way. For $z \in X$, we set

$$
\|d z\|_{\mu, \mathrm{res}}^{2}=\lim _{w \rightarrow z}\left(G_{\mu}(z, w) \cdot|z-w|^{2}\right) .
$$

We call the metric

$$
\mu_{\mathrm{res}}(z)=\frac{i}{2} \cdot \frac{d z \wedge d \bar{z}}{\|d z\|_{\mu, \mathrm{res}}^{2}}
$$

the residual metric associated to $\mu$. If $\mu=\mu_{\text {hyp }}, \mu=\mu_{\text {shyp }}$, or $\mu=\mu_{\text {can }}$, we set

$$
\begin{gathered}
\|\cdot\|_{\mu, \mathrm{res}}=\|\cdot\|_{\text {hyp,res }}, \quad\|\cdot\|_{\mu, \mathrm{res}}=\|\cdot\|_{\text {shyp }, \mathrm{res}}, \quad\|\cdot\|_{\mu, \mathrm{res}}=\|\cdot\|_{\mathrm{can}, \mathrm{res}}, \\
\mu_{\mathrm{res}}=\mu_{\mathrm{hyp}, \mathrm{res}}, \quad \mu_{\mathrm{res}}=\mu_{\mathrm{shyp}, \mathrm{res}}, \quad \mu_{\mathrm{res}}=\mu_{\mathrm{can}, \mathrm{res}},
\end{gathered}
$$




\section{J. JoRgEnSON AND J. KRAmER}

respectively. We recall that the Arakelov metric $\mu_{\text {Ar }}$ is defined as the residual metric associated to the canonical metric $\mu_{\text {can }}$; the corresponding metric on $\Omega_{X}^{1}$ is denoted by $\|\cdot\|_{\text {Ar }}$. In order to be able to compare the metrics $\mu_{\mathrm{can}}$ and $\mu_{\mathrm{Ar}}$, we define the $C^{\infty}$-function $\phi_{\mathrm{Ar}}$ on $X$ by the equation

$$
\mu_{\mathrm{Ar}}=e^{\phi_{\mathrm{Ar}}} \mu_{\mathrm{hyp}} \text {. }
$$

\subsection{Heat kernels and heat traces}

The heat kernel $K_{\mathbb{H}}(t ; z, w)$ on $\mathbb{H}\left(t \in \mathbb{R}_{>0} ; z, w \in \mathbb{H}\right)$ is given by the formula

$$
K_{\mathbb{H}}(t ; z, w)=K_{\mathbb{H}}(t ; \rho)=\frac{\sqrt{2} e^{-t / 4}}{(4 \pi t)^{3 / 2}} \int_{\rho}^{\infty} \frac{u e^{-u^{2} / 4 t}}{\sqrt{\cosh (u)-\cosh (\rho)}} d u,
$$

where $\rho=d_{\mathbb{H}}(z, w)$ denotes the hyperbolic distance between $z$ and $w$. If $z=w$, the previous formula can be shown to be equal to

$$
K_{\mathbb{H}}(t ; z, z)=K_{\mathbb{H}}(t ; 0)=\frac{1}{2 \pi} \int_{0}^{\infty} e^{-\left(r^{2}+1 / 4\right) t} r \tanh (\pi r) d r .
$$

The heat kernel $K_{X}(t ; z, w)$ associated to $X\left(t \in \mathbb{R}_{>0} ; z, w \in X\right)$, respectively the hyperbolic heat kernel $H K_{X}(t ; z, w)$ associated to $X\left(t \in \mathbb{R}_{>0} ; z, w \in X\right)$ is defined by averaging over the elements of $\Gamma$, respectively the elements of $\Gamma$ different from the identity, namely

$$
\begin{aligned}
K_{X}(t ; z, w) & =\sum_{\gamma \in \Gamma} K_{\mathbb{H}}(t ; z, \gamma w), \\
H K_{X}(t ; z, w) & =\sum_{\substack{\gamma \in \Gamma \\
\gamma \neq \mathrm{id}}} K_{\mathbb{H}}(t ; z, \gamma w),
\end{aligned}
$$

respectively. The heat kernel $K_{X}(t ; z, w)$ admits the following spectral representation. Let $\left\{\lambda_{X, n}\right\}$ denote the set of eigenvalues of the hyperbolic Laplacian $\Delta_{X}$, which acts on the space of smooth functions on $X$ with associated orthonormal eigenfunctions $\left\{\varphi_{X, n}\right\}$. Then, for all $z, w \in X$, we have

$$
K_{X}(t ; z, w)=\sum_{n} \varphi_{X, n}(z) \varphi_{X, n}(w) e^{-\lambda_{X, n} t} .
$$

The convergence of this series is uniform and absolute (see [Cha84, p. 112]). Recall that the eigenfunctions can be taken to be real-valued, so there is no need for a complex conjugate over one of the terms.

If $z=w$, we write $K_{X}(t ; z)$ instead of $K_{X}(t ; z, z)$ and $H K_{X}(t ; z)$ instead of $H K_{X}(t ; z, z)$. The hyperbolic heat trace $H \operatorname{Tr} K_{X}(t)\left(t \in \mathbb{R}_{>0}\right)$ is now given by

$$
H \operatorname{Tr} K_{X}(t)=\int_{X} H K_{X}(t ; z) \mu_{\mathrm{hyp}}(z) .
$$

We note that the hyperbolic Green's function $g_{\text {hyp }}(z, w)(z, w \in X ; z \neq w)$ relates in the following way to the heat kernel

$$
g_{\text {hyp }}(z, w)=4 \pi \int_{0}^{\infty}\left(K_{X}(t ; z, w)-\frac{1}{v_{X}}\right) d t .
$$

The hyperbolic Green's function on $\mathbb{H}$ can be defined using the hyperbolic heat kernel, namely through the formula

$$
g_{\mathbb{H}}(z, w)=4 \pi \int_{0}^{\infty} K_{\mathbb{H}}(t ; z, w) d t
$$

As stated in the introduction, explicit formulas were given evaluating $g_{\mathbb{H}}(z, w)$, namely

$$
g_{\mathbb{H}}(z, w)=-\log \left(\left|\frac{z-w}{z-\bar{w}}\right|^{2}\right),
$$




\section{BOUNDS ON CANONICAL GREEN'S FUNCTIONS}

as well as

$$
g_{\mathbb{H}}(z, w)=-\log \left(\tanh ^{2}\left(d_{\mathbb{H}}(z, w) / 2\right)\right)
$$

with $d_{\mathbb{H}}(z, w)$ denoting the hyperbolic distance from $z$ to $w$ (see [Hej83, p. 31], and [Bea95, p. 130]). Both identities will play a role in our work.

\subsection{Selberg's zeta function}

Let $H(\Gamma)$ denote a complete set of representatives of non-conjugate, primitive, hyperbolic elements in $\Gamma$. Denote by $\ell_{\gamma}$ the hyperbolic length of the closed geodesic determined by $\gamma \in H(\Gamma)$ on $X$; it is well known that the equality

$$
|\operatorname{tr}(\gamma)|=2 \cosh \left(\ell_{\gamma} / 2\right)
$$

holds. For $s \in \mathbb{C}, \operatorname{Re}(s)>1$, the Selberg zeta function $Z_{X}(s)$ associated to $X$ is defined via the Euler product expansion

$$
Z_{X}(s)=\prod_{\gamma \in H(\Gamma)} Z_{\gamma}(s), \quad \text { where } Z_{\gamma}(s)=\prod_{n=0}^{\infty}\left(1-e^{-(s+n) \ell_{\gamma}}\right) .
$$

The Selberg zeta function $Z_{X}(s)$ is known to have a meromorphic continuation to all of $\mathbb{C}$ and satisfies a functional equation. As in [JK01], we define the quantity

$$
c_{X}=\lim _{s \rightarrow 1}\left(\frac{Z_{X}^{\prime}}{Z_{X}}(s)-\frac{1}{s-1}\right),
$$

which expresses $c_{X}$ in terms of the hyperbolic heat kernel. From [JK01, Lemma 4.2], we recall the formula

$$
c_{X}=1+\int_{0}^{\infty}\left(H \operatorname{Tr} K_{X}(t)-1\right) d t=\int_{0}^{\infty}\left(H \operatorname{Tr} K_{X}(t)-1+e^{-t}\right) d t .
$$

The quantity $c_{X}$ was studied in detail in [JK01]. Specifically, upper and lower bounds for $c_{X}$ were obtained for a fixed hyperbolic Riemann surface $X$, and these bounds were also studied for surfaces $X_{1}$, which are finite-degree covers of a fixed hyperbolic Riemann surface $X_{0}$. The analysis of $c_{X}$ was extended to the sequence $\left\{X_{0}(N)\right\}$ of hyperbolic modular surfaces in [JK04b, $\left.\S 5\right]$.

\subsection{Heat kernel bounds}

Directly from the integral formula $(2)$ for $K_{\mathbb{H}}(t ; \rho)$, one can prove the following two bounds. First, for any $0<t_{0}<1$, there is a constant $c_{0}>0$ such that for $0<t<t_{0}$, we have the upper bound

$$
K_{\mathbb{H}}(t ; \rho) \leqslant \frac{c_{0}}{4 \pi t} e^{-\rho^{2} /(4 t)}
$$

for all $\rho \geqslant 0$. Second, there is a constant $c_{\infty}>0$ such that, if $t \geqslant t_{0}$, then

$$
K_{\mathbb{H}}(t ; \rho) \leqslant c_{\infty} e^{-t / 4}
$$

for all $\rho \geqslant 0$. Continuing, one also has the bound

$$
K_{X}(t ; z, w) \leqslant \frac{1}{2}\left(K_{X}(t ; z)+K_{X}(t ; w)\right),
$$

which holds for all $t>0$ and all $z, w \in X$. To prove this inequality, observe that for each $n$, we have

$$
\varphi_{X, n}(z) \varphi_{X, n}(w) e^{-\lambda_{X, n} t} \leqslant \frac{1}{2}\left(\varphi_{X, n}^{2}(z) e^{-\lambda_{X, n} t}+\varphi_{X, n}^{2}(w) e^{-\lambda_{X, n} t}\right),
$$

from which the stated bound now follows by summing over all $n$.

More generally, one can use hyperbolic geometry in order to prove an upper bound for $K_{X}(t ; z, w)$. For this, we follow [JL95, Lemma 2.3], in particular displayed formula (2.2) on p. 796, which we now recall in detail. Fix $0<t_{0}<1$, and choose $\delta_{0}$ sufficiently large such that $K_{\mathbb{H}}(t ; \rho)$ 


\section{J. Jorgenson And J. KRAmer}

is a monotone decreasing function of $\rho$ for $\rho>\delta_{0}$ and all $0<t<t_{0}$ (as with the above bounds for $K_{\mathbb{H}}(t ; \rho)$, the verification of the existence of $t_{0}$ and $\delta_{0}$ follows from the integral formula for $\left.K_{\mathbb{H}}(t ; \rho)\right)$. Let $r_{X}$ be any number less than or equal to the injectivity radius of $X$, meaning

$$
r_{X} \leqslant \inf \left\{d_{\mathbb{H}}(z, \gamma z) \mid \gamma \in \Gamma, \gamma \neq \mathrm{id}, z \in X\right\} .
$$

Since $X$ is compact, one can choose $r_{X}>0$. For $\delta>0$ and fixed $z, w \in X$, we define the set

$$
S_{\Gamma}(\delta ; z, w)=\left\{\gamma \in \Gamma \mid d_{\mathbb{H}}(z, \gamma w)<\delta\right\} .
$$

Then, as stated in [JL95, formula (2.2), p. 796], we have the bounds

$$
\sum_{\gamma \in S_{\Gamma}(\delta ; z, w)} K_{\mathbb{H}}\left(t ; d_{\mathbb{H}}(z, \gamma w)\right) \leqslant K_{X}(t ; z, w)
$$

and, for all $0<t<t_{0}$ and $\delta>\delta_{0}$, we have

$$
\begin{aligned}
K_{X}(t ; z, w) \leqslant & \sum_{\gamma \in S_{\Gamma}(\delta ; z, w)} K_{\mathbb{H}}\left(t ; d_{\mathbb{H}}(z, \gamma w)\right)+\frac{\sinh \left(r_{X}\right) \sinh (\delta)}{\sinh ^{2}\left(r_{X} / 2\right)} \cdot K_{\mathbb{H}}(t ; \delta) \\
& +\frac{1}{\sinh ^{2}\left(r_{X} / 2\right)} \int_{\delta-4 r_{X}}^{\infty} K_{\mathbb{H}}(t ; \rho) \sinh \left(\rho+2 r_{X}\right) d \rho
\end{aligned}
$$

The arguments proving these bounds are elementary and we refer the reader to [JL95] for details. We note here that the statement above is obtained through a slight refinement of that given in [JL95], coming from observing that the various hyperbolic discs whose volumes are used to estimate the number of lattice points can be taken to be centered at an orbit point of $w$. As a result, certain estimates above involve $r_{X}$ rather than $2 r_{X}$, as in [JL95]. This refinement is not critical for the analysis here; nonetheless, for the sake of precision, we do quote and then employ this refined result.

\subsection{Certain hyperbolic-geometric invariants}

For the convenience of the reader, we list here certain hyperbolic invariants that appear in our estimates.

The constants $c_{0}, c_{\infty}, t_{0}$, and $\delta_{0}$ appear in the upper bounds for $K_{\mathbb{H}}(t ; \rho)$ and were defined in $\S 2.5$. The constant $r_{X}$ is any number less than or equal to the injectivity radius of $X$, and we take $\delta_{X}$ to be any number such that $\delta_{X}>\max \left\{\delta_{0}, 4 r_{X}+5\right\}>0$. Given $0<t_{0}<1$, we define

$$
C_{X}^{H K}=\max _{z \in X} K_{X}\left(t_{0} ; z\right)
$$

which is finite, since $X$ is compact. Following the arguments in [JL95], it can be shown that

$$
\sup _{z, w \in X} \# S_{\Gamma}(\delta ; z, w) \leqslant \frac{\sinh \left(\delta+r_{X}\right)}{\sinh \left(r_{X}\right)}
$$

where the set $S_{\Gamma}(\delta ; z, w)$ was defined in $\S 2.5$. The smallest non-zero eigenvalue of the hyperbolic Laplacian on $X$ is denoted by $\lambda_{X, 1}$ and the length of the shortest non-zero closed geodesic on $X$ is denoted by $\ell_{X, 0}$. The constant $c_{X}$ is the constant term in the Laurent expansion of the logarithmic derivative of the Selberg zeta function $Z_{X}(s)$ at $s=1$, as defined in $\S 2.4$. Finally, the sup-norm between the canonical and scaled hyperbolic volume forms is defined by

$$
d_{X}=\sup _{z \in X}\left|\frac{\mu_{\text {can }}(z)}{\mu_{\text {shyp }}(z)}\right| .
$$




\section{Bounds on CANONICAL GReEn's FunCtions}

\section{Expressing canonical Green's function using hyperbolic data}

In this section we obtain a closed-form expression for the canonical Green's function in terms of hyperbolic geometry. The main result of this section, Theorem 3.8, expresses $g_{\text {can }}$ in terms of the hyperbolic Green's function $g_{\text {hyp }}$ and analytic functions derived from the hyperbolic heat kernel. The steps in our proof are as follows. First, we derive a general expression relating $g_{\text {can }}$ to $g_{\text {hyp }}$ in terms of various integrals involving $\mu_{\text {can }}$; see Lemma 3.1. Next, we prove an explicit relation between the canonical metric $\mu_{\mathrm{can}}$ and the hyperbolic metric $\mu_{\text {hyp }}$ in terms of the hyperbolic heat kernel; see Theorem 3.4. We then substitute Theorem 3.4 into Lemma 3.1 in order to complete the proof of Theorem 3.8.

Lemma 3.1. With the above notation, we have, for all $z, w \in X$, the formula

$$
\begin{aligned}
g_{\mathrm{hyp}}(z, w)-g_{\mathrm{can}}(z, w)= & \int_{X} g_{\mathrm{hyp}}(z, \zeta) \mu_{\mathrm{can}}(\zeta)+\int_{X} g_{\mathrm{hyp}}(w, \zeta) \mu_{\mathrm{can}}(\zeta) \\
& -\int_{X} \int_{X} g_{\mathrm{hyp}}(\xi, \zeta) \mu_{\mathrm{can}}(\zeta) \mu_{\mathrm{can}}(\xi) .
\end{aligned}
$$

Proof. Let $F_{\mathrm{L}}(z, w)$ (respectively $\left.F_{\mathrm{R}}(z, w)\right)$ denote the left-hand side (respectively right-hand side) of the stated identity. Using the characterizing properties of the Green's functions, one can show directly that we have, for fixed $w \in X$,

$$
d_{z} d_{z}^{c} F_{\mathrm{L}}(z, w)=d_{z} d_{z}^{c} F_{\mathrm{R}}(z, w)=\mu_{\text {shyp }}(z)-\mu_{\mathrm{can}}(z)
$$

and

$$
\int_{X} F_{\mathrm{L}}(z, w) \mu_{\mathrm{can}}(z)=\int_{X} F_{R}(z, w) \mu_{\mathrm{can}}(z)=\int_{X} g_{\mathrm{hyp}}(w, \zeta) \mu_{\mathrm{can}}(\zeta) .
$$

Consequently, $F_{\mathrm{L}}(z, w)=F_{\mathrm{R}}(z, w)$, again for fixed $w$. However, it is obvious that $F_{\mathrm{L}}$ and $F_{\mathrm{R}}$ are symmetric in $z$ and $w$. This completes the proof of the lemma.

Proposition 3.2. With the above notation, we have, for all $z \in X$, the formula

$$
g_{X} \mu_{\text {can }}(z)=\mu_{\text {shyp }}(z)+\frac{1}{2} c_{1}\left(\Omega_{X}^{1},\|\cdot\|_{\text {hyp }, \text { res }}\right)(z) ;
$$

here $\Omega_{X}^{1}$ denotes the canonical line bundle on $X$.

Proof. Let us rewrite the identity in Lemma 3.1 as

$$
g_{\text {hyp }}(z, w)-g_{\text {can }}(z, w)=\phi(z)+\phi(w),
$$

where

$$
\phi(z)=\int_{X} g_{\mathrm{hyp}}(z, \zeta) \mu_{\mathrm{can}}(\zeta)-\frac{1}{2} \int_{X} \int_{X} g_{\mathrm{hyp}}(\xi, \zeta) \mu_{\mathrm{can}}(\zeta) \mu_{\mathrm{can}}(\xi) .
$$

Taking $d_{z} d_{z}^{c}$ in relation (5), we get the equation

$$
\mu_{\text {shyp }}(z)-\mu_{\text {can }}(z)=d_{z} d_{z}^{c} \phi(z) .
$$

On the other hand, we have by definition

$$
\begin{aligned}
& \log \|d z\|_{\text {hyp,res }}^{2}=\lim _{w \rightarrow z}\left(g_{\text {hyp }}(z, w)+\log |z-w|^{2}\right), \\
& \log \|d z\|_{\text {can,res }}^{2}=\lim _{w \rightarrow z}\left(g_{\text {can }}(z, w)+\log |z-w|^{2}\right) .
\end{aligned}
$$

From this we deduce, again using (5),

$$
\log \|d z\|_{\text {hyp }, \mathrm{res}}^{2}-\log \|d z\|_{\text {can,res }}^{2}=\lim _{w \rightarrow z}\left(g_{\text {hyp }}(z, w)-g_{\text {can }}(z, w)\right)=2 \phi(z) .
$$

Now, taking $-d_{z} d_{z}^{c}$ of (7) yields

$$
\mathrm{c}_{1}\left(\Omega_{X}^{1},\|\cdot\|_{\text {hyp,res }}\right)(z)-\mathrm{c}_{1}\left(\Omega_{X}^{1},\|\cdot\|_{\text {can,res }}\right)(z)=-2 d_{z} d_{z}^{c} \phi(z) .
$$




\section{J. JoRgenson And J. KRAmeR}

Combining (6) and (8) leads to

$$
2\left(\mu_{\text {shyp }}(z)-\mu_{\text {can }}(z)\right)=\mathrm{c}_{1}\left(\Omega_{X}^{1},\|\cdot\|_{\text {can,res }}\right)(z)-\mathrm{c}_{1}\left(\Omega_{X}^{1},\|\cdot\|_{\text {hyp }, \text { res }}\right)(z) .
$$

Recalling

$$
\mathrm{c}_{1}\left(\Omega_{X}^{1},\|\cdot\|_{\text {can,res }}\right)(z)=\left(2 g_{X}-2\right) \mu_{\text {can }}(z),
$$

from (9) we derive

$$
\mu_{\text {shyp }}(z)-\mu_{\text {can }}(z)=\frac{2 g_{X}-2}{2} \mu_{\text {can }}(z)-\frac{1}{2} c_{1}\left(\Omega_{X}^{1},\|\cdot\|_{\text {hyp }, \text { res }}\right)(z),
$$

which proves the proposition.

Proposition 3.3. With the above notation, we have the following formula for the first Chern form of $\Omega_{X}^{1}$ with respect to $\|\cdot\|_{\text {hyp,res }}$ :

$$
\mathrm{c}_{1}\left(\Omega_{X}^{1},\|\cdot\|_{\text {hyp,res }}\right)(z)=\frac{1}{2 \pi} \cdot \mu_{\text {hyp }}(z)+\left(\int_{0}^{\infty} \Delta_{X} K_{X}(t ; z) d t\right) \mu_{\text {hyp }}(z) .
$$

Proof. By our definitions, for $z \in X$ we have

$$
\begin{aligned}
\mathrm{c}_{1}\left(\Omega_{X}^{1},\|\cdot\|_{\text {hyp, res }}\right)(z)= & -d_{z} d_{z}^{c} \log \|d z\|_{\text {hyp,res }}^{2}=-d_{z} d_{z}^{c} \lim _{w \rightarrow z}\left(g_{\text {hyp }}(z, w)+\log |z-w|^{2}\right) \\
= & -d_{z} d_{z}^{c} \lim _{w \rightarrow z}\left(4 \pi \int_{0}^{\infty}\left(K_{X}(t ; z, w)-\frac{1}{v_{X}}\right) d t+\log |z-w|^{2}\right) \\
= & -d_{z} d_{z}^{c} \lim _{w \rightarrow z}\left(4 \pi \int_{0}^{\infty} K_{\mathbb{H}}(t ; z, w) d t+\log |z-w|^{2}\right) \\
& -d_{z} d_{z}^{c} \lim _{w \rightarrow z}\left(4 \pi \int_{0}^{\infty}\left(\sum_{\gamma \in \Gamma} K_{\mathbb{H}}(t ; z, \gamma w)-\frac{1}{v_{X}}\right) d t\right) .
\end{aligned}
$$

Using the formula for the Green's function $g_{\mathbb{H}}(z, w)$ on $\mathbb{H}$, for the first summand in the latter sum we obtain

$$
\begin{aligned}
A & =-d_{z} d_{z}^{c} \lim _{w \rightarrow z}\left(4 \pi \int_{0}^{\infty} K_{\mathbb{H}}(t ; z, w) d t+\log |z-w|^{2}\right) \\
& =-d_{z} d_{z}^{c} \lim _{w \rightarrow z}\left(g_{\mathbb{H}}(z, w)+\log |z-w|^{2}\right) \\
& =-d_{z} d_{z}^{c} \log |z-\bar{z}|^{2}=-\frac{2 i}{2 \pi} \partial_{z} \bar{\partial}_{z} \log (z-\bar{z}) \\
& =\frac{i}{\pi} \partial_{z} \frac{d \bar{z}}{z-\bar{z}}=-\frac{i}{\pi} \cdot \frac{d z \wedge d \bar{z}}{(z-\bar{z})^{2}} \\
& =-\frac{i}{\pi} \cdot \frac{d z \wedge d \bar{z}}{(2 i \operatorname{Im}(z))^{2}}=\frac{1}{2 \pi} \cdot \mu_{\text {hyp }}(z) .
\end{aligned}
$$

For the second summand we obtain

$$
\begin{aligned}
B & =-d_{z} d_{z}^{c} \lim _{w \rightarrow z}\left(4 \pi \int_{0}^{\infty}\left(\sum_{\substack{\gamma \in \Gamma \\
\gamma \neq \mathrm{id}}} K_{\mathbb{H}}(t ; z, \gamma w)-\frac{1}{v_{X}}\right) d t\right) \\
& =-4 \pi d_{z} d_{z}^{c} \int_{0}^{\infty}\left(\sum_{\substack{\gamma \in \Gamma \\
\gamma \neq \mathrm{id}}} K_{\mathbb{H}}(t ; z, \gamma z)-\frac{1}{v_{X}}\right) d t .
\end{aligned}
$$




\section{Bounds on CANONiCAL GREen's FunCtions}

Since the latter integral converges absolutely, as does the integral of derivatives of the integrand, we are allowed to interchange differentiation and integration; this gives

$$
\begin{aligned}
B & =-4 \pi \int_{0}^{\infty} d_{z} d_{z}^{c}\left(\sum_{\substack{\gamma \in \Gamma \\
\gamma \neq \mathrm{id}}} K_{\mathbb{H}}(t ; z, \gamma z)-\frac{1}{v_{X}}\right) d t \\
& =-4 \pi \int_{0}^{\infty} \sum_{\substack{\gamma \in \Gamma \\
\gamma \neq \mathrm{id}}} d_{z} d_{z}^{c} K_{\mathbb{H}}(t ; z, \gamma z) d t .
\end{aligned}
$$

The claimed formula then follows, since $K_{\mathbb{H}}(t ; z, z)$ is independent of $z$, and recalling the identity

$$
-4 \pi d_{z} d_{z}^{c} f(z)=\left(\Delta_{X} f(z)\right) \mu_{\mathrm{hyp}}(z)
$$

for any smooth function $f$ on $X$.

Theorem 3.4. With the above notation, we have, for all $z \in X$, the formula

$$
\mu_{\text {can }}(z)=\mu_{\text {shyp }}(z)+\frac{1}{2 g_{X}}\left(\int_{0}^{\infty} \Delta_{X} K_{X}(t ; z) d t\right) \mu_{\text {hyp }}(z) .
$$

Proof. We simply have to combine Propositions 3.2 and 3.3, and to use that

$$
\frac{1}{g_{X}}+\frac{v_{X}}{4 \pi g_{X}}=1
$$

Lemma 3.5. For all $z \in X$, let $H(z)$ be defined by

$$
H(z)=\int_{0}^{\infty}\left(H K_{X}(t ; z)-\frac{1}{v_{X}}\right) d t-\frac{c_{X}-1}{v_{X}} .
$$

Then, $H(z)$ is uniquely characterized by satisfying the integral formula

$$
\int_{X} H(z) \mu_{\mathrm{hyp}}(z)=0
$$

and the differential equation

$$
\Delta_{X} H(z)=\int_{0}^{\infty} \Delta_{X} K_{X}(t ; z) d t
$$

Proof. Concerning the integral equation, note that, by interchanging the order of integration, we have

$$
\begin{aligned}
\int_{X} H(z) \mu_{\mathrm{hyp}}(z) & =\int_{X}\left(\int_{0}^{\infty}\left(H K_{X}(t ; z)-\frac{1}{v_{X}}\right) d t-\frac{c_{X}-1}{v_{X}}\right) \mu_{\mathrm{hyp}}(z) \\
& =\int_{0}^{\infty}\left(H \operatorname{Tr} K_{X}(t)-1\right) d t-\left(c_{X}-1\right)=0
\end{aligned}
$$

where the last equality follows from formula (4), given in $\S 2.4$. As for the differential equation, note that for any $z \in X$, we have

$$
H K_{X}(t ; z)=K_{X}(t ; z)-K_{\mathbb{H}}(t, 0) .
$$

Since $K_{\mathbb{H}}(t, 0)$ and $\left(c_{X}-1\right) / v_{X}$ are annihilated by $\Delta_{X}$, the result follows.

Lemma 3.6. With the above notation, we have, for all $z \in X$, the formula

$$
\int_{X} g_{\mathrm{hyp}}(z, \zeta) \mu_{\mathrm{can}}(\zeta)=\frac{2 \pi}{g_{X}} H(z)
$$




\section{J. Jorgenson And J. KRAmer}

Proof. Using Theorem 3.4, we have

$$
\begin{aligned}
\int_{X} g_{\mathrm{hyp}}(z, \zeta) \mu_{\mathrm{can}}(\zeta) & =\int_{X} g_{\mathrm{hyp}}(z, \zeta)\left(\mu_{\mathrm{shyp}}(\zeta)+\frac{1}{2 g_{X}}\left(\int_{0}^{\infty} \Delta_{X} K_{X}(t ; \zeta) d t\right) \mu_{\mathrm{hyp}}(\zeta)\right) \\
& =\frac{1}{2 g_{X}} \int_{X} g_{\mathrm{hyp}}(z, \zeta)\left(\int_{0}^{\infty} \Delta_{X} K_{X}(t ; \zeta) d t\right) \mu_{\mathrm{hyp}}(\zeta) \\
& =\frac{1}{2 g_{X}} \int_{X} g_{\mathrm{hyp}}(z, \zeta) \Delta_{X} H(\zeta) \mu_{\mathrm{hyp}}(\zeta),
\end{aligned}
$$

where the last equality follows from Lemma 3.5. Using the integral formula in Lemma 3.5, the assertion is proved by using that $g_{\text {hyp }}$ inverts the operator $-d d^{c}$ on the space of functions whose integral is zero.

LEMmA 3.7. With the above notation, we have the formula

$$
\int_{X} \int_{X} g_{\mathrm{hyp}}(\xi, \zeta) \mu_{\mathrm{can}}(\zeta) \mu_{\mathrm{can}}(\xi)=\frac{\pi}{g_{X}^{2}} \int_{X} H(\xi) \Delta_{X} H(\xi) \mu_{\mathrm{hyp}}(\xi) .
$$

Proof. Using Lemma 3.6, we have

$$
\int_{X} \int_{X} g_{\text {hyp }}(\xi, \zeta) \mu_{\text {can }}(\zeta) \mu_{\text {can }}(\xi)=\frac{2 \pi}{g_{X}} \int_{X} H(\xi) \mu_{\text {can }}(\xi)
$$

We now employ Theorem 3.4, which gives

$$
\begin{aligned}
\int_{X} H(\xi) \mu_{\mathrm{can}}(\xi) & =\int_{X} H(\xi)\left(\mu_{\text {shyp }}(\xi)+\frac{1}{2 g_{X}}\left(\int_{0}^{\infty} \Delta_{X} K_{X}(t ; \xi) d t\right) \mu_{\mathrm{hyp}}(\xi)\right) \\
& =\frac{1}{2 g_{X}} \int_{X} H(\xi)\left(\int_{0}^{\infty} \Delta_{X} K_{X}(t ; \xi) d t\right) \mu_{\mathrm{hyp}}(\xi),
\end{aligned}
$$

where we have used the integral equation from Lemma 3.5 to obtain the last equality. The result follows by using the differential equation from Lemma 3.5.

THEOREM 3.8. With the above notation, we have the formula

$$
g_{\mathrm{can}}(z, w)-g_{\mathrm{hyp}}(z, w)=\phi_{X}(z)+\phi_{X}(w),
$$

where

$$
\phi_{X}(z)=\frac{2 \pi}{g_{X}} H(z)-\frac{\pi}{2 g_{X}^{2}} \int_{X} H(\xi) \Delta_{X} H(\xi) \mu_{\text {hyp }}(\xi) .
$$

Proof. The proof is obtained by combining Lemmas 3.1, 3.6 and 3.7.

Remark 3.9. Recall from $\S 2.3$ that the hyperbolic Green's function $g_{\mathrm{hyp}}$ is simply expressed in terms of the hyperbolic heat kernel. Together with the definition of $H(z)$ given in Lemma 3.5, the main result in Theorem 3.8 then states a closed form expression for the canonical Green's function $g_{\text {can }}$ using the hyperbolic heat kernel. By comparison, note that the analysis in [Jor90] relied on an evaluation of the canonical Green's function in terms of the classical Riemann theta function; see [Jor90], in particular Proposition 2.4 and the preceding computations. Consequently, we now have a complete, closed-form expression for the Riemann theta function in terms of the hyperbolic heat kernel. A potentially fascinating study would be to explore this relation further, either from the point of view of obtaining results in hyperbolic geometry from the algebraic geometry of the theta function, or conversely.

\section{Bounds of various hyperbolic data}

We now work from Theorem 3.8 and obtain bounds for the canonical Green's function for a fixed surface $X$. First, we study bounds for the hyperbolic Green's function, which we derive using the heat 


\section{BOUNDS ON CANONICAL GREEN'S FUNCTIONS}

kernel bound stated in $\S 2.5$; these bounds are given in Theorem 4.5. Next, we estimate the function $\phi_{X}$ in Theorem 3.8; these estimates are given in Corollary 4.6 and Proposition 4.7. After this, the bounds we seek for the canonical Green's function are immediate and are stated in Theorems 4.8 and 4.9. As we will see in the next section, the explicit nature of these bounds are such that we can easily determine the behavior of the estimates through covers and for sequences of hyperbolic modular curves.

LemmA 4.1. Let $t_{0}$ and $C_{X}^{H K}$ be as in $\S \S 2.5$ and 2.6. For any $\varepsilon>0$ and $z, w \in X$, we then have the following estimate involving the eigenfunctions $\varphi_{X, n}$ of the hyperbolic Laplacian

$$
\sum_{0 \leqslant \lambda_{X, n}<\varepsilon}\left|\varphi_{X, n}(z) \varphi_{X, n}(w)\right| \leqslant C_{X}^{H K} \cdot e^{\varepsilon t_{0}} .
$$

Proof. First observe that for each $n$, we have

$$
\left|\varphi_{X, n}(z) \varphi_{X, n}(w)\right| \leqslant \frac{1}{2}\left(\varphi_{X, n}^{2}(z)+\varphi_{X, n}^{2}(w)\right) ;
$$

hence, it suffices to prove the claim when $z=w$. For this, we note that $e^{-\lambda_{X, n} t_{0}} \cdot e^{\varepsilon t_{0}} \geqslant 1$, provided that $\lambda_{X, n}<\varepsilon$. Therefore, we find

$$
\sum_{0 \leqslant \lambda_{X, n}<\varepsilon} \varphi_{X, n}^{2}(z) \leqslant \sum_{0 \leqslant \lambda_{X, n}<\varepsilon} \varphi_{X, n}^{2}(z) e^{-\lambda_{X, n} t_{0}} \cdot e^{\varepsilon t_{0}} \leqslant e^{\varepsilon t_{0}} \cdot K_{X}\left(t_{0} ; z\right) \leqslant C_{X}^{H K} \cdot e^{\varepsilon t_{0}},
$$

which proves the claim.

Lemma 4.2. Let $c_{0}, c_{\infty}, t_{0}, r_{X}, \delta_{X}$, and $C_{X}^{H K}$ be as in $\S \S 2.5$ and 2.6. For any $\delta \geqslant \delta_{X}, \varepsilon>0$, and $z, w \in X$, let

$$
K_{X}^{\varepsilon, \delta}(t ; z, w)=K_{X}(t ; z, w)-\sum_{0 \leqslant \lambda_{X, n}<\varepsilon} \varphi_{X, n}(z) \varphi_{X, n}(w) e^{-\lambda_{X, n} t}-\sum_{\gamma \in S_{\Gamma}(\delta ; z, w)} K_{\mathbb{H}}\left(t ; d_{\mathbb{H}}(z, \gamma w)\right) .
$$

Then, we have the following bounds:

(a) if $0<t<t_{0}$, then

$$
\left|K_{X}^{\varepsilon, \delta}(t ; z, w)\right| \leqslant C_{X}^{H K} \cdot e^{\varepsilon t_{0}}+\frac{c_{0} \sinh \left(r_{X}\right) \sinh (\delta)}{8 \delta^{2} \sinh ^{2}\left(r_{X} / 2\right)}+\frac{c_{0} e^{2 r_{X}}}{2 \pi \sinh ^{2}\left(r_{X} / 2\right)} ;
$$

(b) if $t \geqslant t_{0}$, then

$$
\left|K_{X}^{\varepsilon, \delta}(t ; z, w)\right| \leqslant C_{X}^{H K} \cdot e^{-\varepsilon\left(t-t_{0}\right)}+\frac{c_{\infty} \sinh \left(\delta+r_{X}\right)}{\sinh \left(r_{X}\right)} e^{-t / 4} .
$$

Proof. To prove part (a), we first use the triangle inequality to write

$$
\left|K_{X}^{\varepsilon, \delta}(t ; z, w)\right| \leqslant \sum_{0 \leqslant \lambda_{X, n}<\varepsilon}\left|\varphi_{X, n}(z) \varphi_{X, n}(w)\right| e^{-\lambda_{X, n} t}+\sum_{\gamma \notin S_{\Gamma}(\delta ; z, w)} K_{\mathbb{H}}\left(t ; d_{\mathbb{H}}(z, \gamma w)\right) .
$$

By Lemma 4.1, the first summand is bounded by $C_{X}^{H K} \cdot e^{\varepsilon t_{0}}$. As for the second summand, we proceed by using the heat kernel estimates from $\S 2.5$, namely the bounds

$$
\begin{aligned}
\sum_{\gamma \notin S_{\Gamma}(\delta ; z, w)} K_{\mathbb{H}}\left(t ; d_{\mathbb{H}}(z, \gamma w)\right) \leqslant & \frac{\sinh \left(r_{X}\right) \sinh (\delta)}{\sinh ^{2}\left(r_{X} / 2\right)} \cdot K_{\mathbb{H}}(t ; \delta) \\
& +\frac{1}{\sinh ^{2}\left(r_{X} / 2\right)} \int_{\delta-4 r_{X}}^{\infty} K_{\mathbb{H}}(t ; \rho) \sinh \left(\rho+2 r_{X}\right) d \rho .
\end{aligned}
$$

Trivially, the lower bound for the sum in question is zero, since each term in the series is positive. Since $0<t<t_{0}<1$, we can use the bound

$$
K_{\mathbb{H}}(t ; \delta) \leqslant \frac{c_{0}}{4 \pi t} e^{-\delta^{2} /(4 t)},
$$




\section{J. Jorgenson AND J. KRAmer}

which gives

$$
\frac{\sinh \left(r_{X}\right) \sinh (\delta)}{\sinh ^{2}\left(r_{X} / 2\right)} \cdot K_{\mathbb{H}}(t ; \delta) \leqslant \frac{c_{0} \sinh \left(r_{X}\right) \sinh (\delta)}{\sinh ^{2}\left(r_{X} / 2\right)} \cdot \frac{1}{4 \pi t} e^{-\delta^{2} /(4 t)} .
$$

It is elementary to compute that the maximum of $e^{-a / t} / t$, as a function of $t>0$ and fixed $a>0$, occurs when $t=a$, yielding the maximum value of $e^{-1} / a$. Therefore, taking $a=\delta^{2} / 4$, we get

$$
\frac{c_{0} \sinh \left(r_{X}\right) \sinh (\delta)}{\sinh ^{2}\left(r_{X} / 2\right)} \cdot \frac{1}{4 \pi t} e^{-\delta^{2} /(4 t)} \leqslant \frac{c_{0} \sinh \left(r_{X}\right) \sinh (\delta)}{\sinh ^{2}\left(r_{X} / 2\right)} \cdot \frac{1}{\pi \delta^{2}} e^{-1} \leqslant \frac{c_{0} \sinh \left(r_{X}\right) \sinh (\delta)}{8 \delta^{2} \sinh ^{2}\left(r_{X} / 2\right)},
$$

using that $\pi e>8$; thus, we have computed the second term in the stated upper bound. For the last term, we use the stated upper bound for $K_{\mathbb{H}}(t ; \rho)$ together with the trivial estimate $\sinh (x) \leqslant e^{x} / 2$ in order to write

$$
\frac{1}{\sinh ^{2}\left(r_{X} / 2\right)} \int_{\delta-4 r_{X}}^{\infty} K_{\mathbb{H}}(t ; \rho) \sinh \left(\rho+2 r_{X}\right) d \rho \leqslant \frac{c_{0} e^{2 r_{X}}}{8 \pi t \cdot \sinh ^{2}\left(r_{X} / 2\right)} \int_{\delta-4 r_{X}}^{\infty} e^{-\rho^{2} /(4 t)+\rho} d \rho .
$$

Over the specified limits of integration, we have that $\rho^{2} \geqslant \rho\left(\delta-4 r_{X}\right)$, so then

$$
e^{-\rho^{2} /(4 t)+\rho} \leqslant e^{-\rho\left(\delta-4 r_{X}\right) /(4 t)+\rho}=e^{-\rho\left(\delta-4 r_{X}-4 t\right) /(4 t)} .
$$

By assumption, $\delta \geqslant \delta_{X}>4 r_{X}+5$, so then for $0<t<t_{0}<1$, we have that $\delta-4 r_{X}-4 t>1$, hence the exponential functions $e^{-\rho^{2} /(4 t)+\rho}$ are integrable for all $0<t<t_{0}$ near infinity. With this, we then have

$$
\int_{\delta-4 r_{X}}^{\infty} e^{-\rho^{2} /(4 t)+\rho} d \rho \leqslant \int_{\delta-4 r_{X}}^{\infty} e^{-\rho\left(\delta-4 r_{X}-4 t\right) /(4 t)} d \rho=\frac{4 t}{\delta-4 r_{X}-4 t} e^{-\left(\delta-4 r_{X}\right)\left(\delta-4 r_{X}-4 t\right) /(4 t)} .
$$

Since $\delta-4 r_{X}-4 t>1$, we have $\delta-4 r_{X}>1$, so then

$$
\frac{4 t}{\delta-4 r_{X}-4 t} e^{-\left(\delta-4 r_{X}\right)\left(\delta-4 r_{X}-4 t\right) /(4 t)} \leqslant 4 t \cdot e^{-\left(\delta-4 r_{X}\right)\left(\delta-4 r_{X}-4 t\right) /(4 t)} \leqslant 4 t .
$$

Summing up, we find

$$
\frac{1}{\sinh ^{2}\left(r_{X} / 2\right)} \int_{\delta-4 r_{X}}^{\infty} K_{\mathbb{H}}(t ; \rho) \sinh \left(\rho+2 r_{X}\right) d \rho \leqslant \frac{c_{0} e^{2 r_{X}}}{2 \pi \sinh ^{2}\left(r_{X} / 2\right)},
$$

which completes the proof of part (a).

We now prove part (b). To begin, we use the spectral decomposition of the heat kernel and the triangle inequality to get

$$
\left|K_{X}^{\varepsilon, \delta}(t ; z, w)\right| \leqslant \sum_{\lambda_{X, n} \geqslant \varepsilon}\left|\varphi_{X, n}(z) \varphi_{X, n}(w)\right| e^{-\lambda_{X, n} t}+\sum_{\gamma \in S_{\Gamma}(\delta ; z, w)} K_{\mathbb{H}}\left(t ; d_{\mathbb{H}}(z, \gamma w)\right) .
$$

From $\S 2.6$, we then have

$$
\sum_{\gamma \in S_{\Gamma}(\delta ; z, w)} K_{\mathbb{H}}\left(t ; d_{\mathbb{H}}(z, \gamma w)\right) \leqslant \# S_{\Gamma}(\delta ; z, w) \cdot \sup _{\eta \in[0, \delta]} K_{\mathbb{H}}(t ; \eta) \leqslant \frac{c_{\infty} \sinh \left(\delta+r_{X}\right)}{\sinh \left(r_{X}\right)} e^{-t / 4},
$$

which yields one of the terms in the stated upper bound. For the other term, we note that

$$
\sum_{\lambda_{X, n} \geqslant \varepsilon}\left|\varphi_{X, n}(z) \varphi_{X, n}(w)\right| e^{-\lambda_{X, n} t} \leqslant \frac{1}{2}\left(\sum_{\lambda_{X, n} \geqslant \varepsilon} \varphi_{X, n}^{2}(z) e^{-\lambda_{X, n} t}+\sum_{\lambda_{X, n} \geqslant \varepsilon} \varphi_{X, n}^{2}(w) e^{-\lambda_{X, n} t}\right),
$$

so it suffices to prove that

$$
\sum_{\lambda_{X, n} \geqslant \varepsilon} \varphi_{X, n}^{2}(z) e^{-\lambda_{X, n} t} \leqslant C_{X}^{H K} \cdot e^{-\varepsilon\left(t-t_{0}\right)}
$$




\section{BOUNDS ON CANONICAL GREEN'S FUNCTIONS}

For this, we consider the function

$$
h(t ; z)=e^{\varepsilon t} \cdot \sum_{\lambda_{X, n} \geqslant \varepsilon} \varphi_{X, n}^{2}(z) e^{-\lambda_{X, n} t} .
$$

For fixed $z \in X$, the function $h(t ; z)$ is monotone decreasing in $t$ for all $t>0$. In particular, we then have

$$
h(t ; z) \leqslant h\left(t_{0} ; z\right)=e^{\varepsilon t_{0}} \cdot \sum_{\lambda_{X, n} \geqslant \varepsilon} \varphi_{X, n}^{2}(z) e^{-\lambda_{X, n} t_{0}} \leqslant e^{\varepsilon t_{0}} \cdot K_{X}\left(t_{0} ; z\right) \leqslant C_{X}^{H K} \cdot e^{\varepsilon t_{0}} .
$$

Therefore, we end up with

$$
0 \leqslant \sum_{\lambda_{X, n} \geqslant \varepsilon} \varphi_{X, n}^{2}(z) e^{-\lambda_{X, n} t}=e^{-\varepsilon t} \cdot h(t ; z) \leqslant e^{-\varepsilon t} \cdot C_{X}^{H K} \cdot e^{\varepsilon t_{0}}=C_{X}^{H K} \cdot e^{-\varepsilon\left(t-t_{0}\right)} .
$$

With all this, part (b) is proved.

Remark 4.3. If required, the estimates in Lemma 4.2 could be enhanced to reflect the role played by $\delta$. For example, the estimates for $0<t<t_{0}$ can be easily improved so that the upper bound approaches zero as $\delta$ increases. However, rather than weigh down the above estimates any further, we choose to underplay the role of $\delta$ solely because further bounds are not needed in the present article.

Lemma 4.4. For any $z, w \in \mathbb{H}$ with $d_{\mathbb{H}}(z, w) \in[a, b]$, we have the estimate

$$
\left|g_{\mathbb{H}}(z, w)\right| \leqslant \max \left\{\left|\log \left(\tanh ^{2}(a / 2)\right)\right|,\left|\log \left(\tanh ^{2}(b / 2)\right)\right|\right\} .
$$

Proof. From [Bea95, p. 130], we have

$$
g_{\mathbb{H}}(z, w)=-\log \left(\tanh ^{2}\left(d_{\mathbb{H}}(z, w) / 2\right)\right) .
$$

The function $\tanh (u)$ is monotone increasing for $u>0$, so its maximum and minimum for $u \in[a, b]$ occur at the boundary, from which the lemma follows.

Theorem 4.5. Let $c_{0}, c_{\infty}, t_{0}, r_{X}, \delta_{X}$, and $C_{X}^{H K}$ be as in $\S \S 2.5$ and 2.6. For any $\delta>0, \varepsilon>0$, and $z, w \in X$, we then have the estimate

$$
\left|g_{\mathrm{hyp}}(z, w)-\sum_{\gamma \in S_{\Gamma}(\delta ; z, w)} g_{\mathbb{H}}(z, \gamma w)-\sum_{0<\lambda_{X, n}<\varepsilon} \frac{4 \pi}{\lambda_{X, n}} \varphi_{X, n}(z) \varphi_{X, n}(w)\right| \leqslant B_{X, \varepsilon, \delta},
$$

where

$$
B_{X, \varepsilon, \delta}=\left\{\begin{array}{lc}
4 \pi\left(C_{X}^{H K} \cdot e^{\varepsilon t_{0}}+\frac{c_{0} \sinh \left(r_{X}\right) \sinh (\delta)}{8 \delta^{2} \sinh ^{2}\left(r_{X} / 2\right)}+\frac{c_{0} e^{2 r_{X}}}{2 \pi \sinh ^{2}\left(r_{X} / 2\right)}+\frac{4 c_{\infty} \sinh \left(\delta+r_{X}\right)}{\sinh \left(r_{X}\right)}+\frac{C_{X}^{H K}}{\varepsilon}\right), \\
4 \pi\left(C_{X}^{H K} \cdot e^{\varepsilon t_{0}}+\frac{c_{0} \sinh \left(r_{X}\right) \sinh \left(\delta_{X}\right)}{8 \delta_{X}^{2} \sinh ^{2}\left(r_{X} / 2\right)}+\frac{c_{0} e^{2 r_{X}}}{2 \pi \sinh ^{2}\left(r_{X} / 2\right)}+\frac{4 c_{\infty} \sinh \left(\delta_{X}+r_{X}\right)}{\sinh \left(r_{X}\right)}+\frac{C_{X}^{H K}}{\varepsilon}\right) \\
\quad+\frac{\sinh \left(\delta_{X}+r_{X}\right)}{\sinh \left(r_{X}\right)} \max \left\{\left|\log \left(\tanh ^{2}(\delta / 2)\right)\right|,\left|\log \left(\tanh ^{2}\left(\delta_{X} / 2\right)\right)\right|\right\}, & \text { if } \delta \leqslant \delta_{X} .
\end{array}\right.
$$

Proof. By the definition of $K_{X}^{\varepsilon, \delta}(t ; z, w)$ given in Lemma 4.2, we have

$$
g_{\text {hyp }}(z, w)-\sum_{0<\lambda_{X, n}<\varepsilon} \frac{4 \pi}{\lambda_{X, n}} \varphi_{X, n}(z) \varphi_{X, n}(w)-\sum_{\gamma \in S_{\Gamma}(\delta ; z, w)} g_{\mathbb{H}}(z, \gamma w)=4 \pi \int_{0}^{\infty} K_{X}^{\varepsilon, \delta}(t ; z, w) d t .
$$




\section{J. JoRgEnSON AND J. KRAmER}

If $\delta>\delta_{X}$, the result immediately follows from integrating the bounds from Lemma 4.2, taking into account the decomposition

$$
\int_{0}^{\infty}\left|K_{X}^{\varepsilon, \delta}(t ; z, w)\right| d t=\int_{0}^{t_{0}}\left|K_{X}^{\varepsilon, \delta}(t ; z, w)\right| d t+\int_{t_{0}}^{\infty}\left|K_{X}^{\varepsilon, \delta}(t ; z, w)\right| d t
$$

On the other hand, if $\delta \leqslant \delta_{X}$, we simply write

$$
K_{X}^{\varepsilon, \delta}(t ; z, w)=K_{X}^{\varepsilon, \delta_{X}}(t ; z, w)+\sum_{\gamma \in S_{\Gamma}\left(\delta_{X} ; z, w\right) \backslash S_{\Gamma}(\delta ; z, w)} K_{\mathbb{H}}\left(t ; d_{\mathbb{H}}(z, \gamma w)\right) .
$$

Then, taking absolute values and using the triangle inequality, the integral over $\left|K_{X}^{\varepsilon, \delta_{X}}(t ; z, w)\right|$ is estimated as in the previous case using Lemma 4.2, but with $\delta$ replaced by $\delta_{X}$, while the remaining sum is estimated using Lemma 4.3 together with the bound

$$
\#\left(S_{\Gamma}\left(\delta_{X} ; z, w\right) \backslash S_{\Gamma}(\delta ; z, w)\right) \leqslant \# S_{\Gamma}\left(\delta_{X} ; z, w\right) \leqslant \frac{\sinh \left(\delta_{X}+r_{X}\right)}{\sinh \left(r_{X}\right)} .
$$

The proof of the theorem is now complete.

Corollary 4.6. Let $\lambda_{X, 1}$ and $\ell_{X, 0}$ be as in $\S 2.6$, and put

$$
F(z)=\int_{0}^{\infty}\left(H K_{X}(t ; z)-\frac{1}{v_{X}}\right) d t \quad(z \in X) .
$$

For any $\varepsilon \in\left(0, \lambda_{X, 1}\right)$ and $\delta \in\left(0, \ell_{X, 0}\right)$, we then have the estimate

$$
\sup _{z \in X}|F(z)| \leqslant \frac{B_{X, \varepsilon, \delta}}{4 \pi}
$$

where $B_{X, \varepsilon, \delta}$ is as in Theorem 4.5.

Proof. The result follows immediately from the argument given in the proof of Theorem 4.5, taking into account that for the stated choices of $\varepsilon$ and $\delta$, we have

$$
H K_{X}(t ; z)-\frac{1}{v_{X}}=K_{X}^{\varepsilon, \delta}(t ; z, z) .
$$

Proposition 4.7. Let $\lambda_{X, 1}$ and $d_{X}$ be as in $\S 2.6$, and $H(z)$ as in Lemma 3.5. For any Riemann surface $X$ of genus $g_{X}>1$, we then have the estimate

$$
0 \leqslant \frac{\pi}{2 g_{X}^{2}} \int_{X} H(z) \Delta_{X} H(z) \mu_{\mathrm{hyp}}(z) \leqslant \frac{\pi\left(d_{X}+1\right)^{2} v_{X}}{2 g_{X}^{2} \lambda_{X, 1}} .
$$

Proof. With $H(z)$ as in Lemma 3.5, we have as in Corollary 4.6

$$
F(z)=\int_{0}^{\infty}\left(H K_{X}(t ; z)-\frac{1}{v_{X}}\right) d t=H(z)+\frac{c_{X}-1}{v_{X}} .
$$

It is elementary to show that

$$
\int_{X} H(z) \Delta_{X} H(z) \mu_{\mathrm{hyp}}(z)=\int_{X} F(z) \Delta_{X} F(z) \mu_{\mathrm{hyp}}(z),
$$

since

$$
\Delta_{X} F(z)=\Delta_{X} H(z) \text { and } \int_{X} \Delta_{X} F(z) \mu_{\text {hyp }}(z)=0 .
$$

Therefore, it suffices to prove that

$$
0 \leqslant \int_{X} F(z) \Delta_{X} F(z) \mu_{\text {hyp }}(z) \leqslant \frac{\left(d_{X}+1\right)^{2} v_{X}}{\lambda_{X, 1}},
$$

which is precisely the statement from [JK04b, Proposition 4.1], which we refer to for further details. 


\section{Bounds on CANONICAL GREEN'S FUnCTIONS}

Theorem 4.8. Let $\lambda_{X, 1}, \ell_{X, 0}, c_{X}$, and $d_{X}$ be as in $\S 2.6$. For any $\varepsilon \in\left(0, \lambda_{X, 1}\right), \delta \in\left(0, \ell_{X, 0}\right)$, and $z, w \in X$, we then have the estimate

$$
\left|g_{\text {can }}(z, w)-g_{\text {hyp }}(z, w)\right| \leqslant \frac{B_{X, \varepsilon, \delta}}{g_{X}}+\frac{4 \pi\left|c_{X}-1\right|}{g_{X} v_{X}}+\frac{\pi\left(d_{X}+1\right)^{2} v_{X}}{g_{X}^{2} \lambda_{X, 1}},
$$

where $B_{X, \varepsilon, \delta}$ is as in Theorem 4.5.

Proof. By combining Theorem 3.8 and Proposition 4.7, we get

$$
\left|g_{\text {can }}(z, w)-g_{\text {hyp }}(z, w)\right| \leqslant \frac{4 \pi}{g_{X}} \sup _{z \in X}|H(z)|+\frac{\pi\left(d_{X}+1\right)^{2} v_{X}}{g_{X}^{2} \lambda_{X, 1}} .
$$

By the definition of $H(z)$ and $F(z)$, we now derive from Corollary 4.6

$$
\sup _{z \in X}|H(z)| \leqslant \sup _{z \in X}|F(z)|+\frac{\left|c_{X}-1\right|}{v_{X}} \leqslant \frac{B_{X, \varepsilon, \delta}}{4 \pi}+\frac{\left|c_{X}-1\right|}{v_{X}} .
$$

By combining the above estimates, the theorem is proved.

Theorem 4.9. Let $\lambda_{X, 1}, \ell_{X, 0}, c_{X}$, and $d_{X}$ be as in $\S 2.6$. For any $\varepsilon \in\left(0, \lambda_{X, 1}\right), \delta \in\left(0, \ell_{X, 0}\right)$, and $z, w \in X$, we then have the estimate

$$
\left|g_{\operatorname{can}}(z, w)-\sum_{\gamma \in S_{\Gamma}(\delta ; z, w)} g_{\mathbb{H}}(z, \gamma w)\right| \leqslant A_{X, \varepsilon, \delta},
$$

where

$$
A_{X, \varepsilon, \delta}=B_{X, \varepsilon, \delta}+\frac{B_{X, \varepsilon, \delta}}{g_{X}}+\frac{4 \pi\left|c_{X}-1\right|}{g_{X} v_{X}}+\frac{\pi\left(d_{X}+1\right)^{2} v_{X}}{g_{X}^{2} \lambda_{X, 1}}
$$

with $B_{X, \varepsilon, \delta}$ as in Theorem 4.5 .

Proof. Since

$$
\begin{aligned}
\left|g_{\mathrm{can}}(z, w)-\sum_{\gamma \in S_{\Gamma}(\delta ; z, w)} g_{\mathbb{H}}(z, \gamma w)\right| \leqslant & \left|g_{\mathrm{can}}(z, w)-g_{\mathrm{hyp}}(z, w)\right| \\
& +\left|g_{\mathrm{hyp}}(z, w)-\sum_{\gamma \in S_{\Gamma}(\delta ; z, w)} g_{\mathbb{H}}(z, \gamma w)\right|,
\end{aligned}
$$

the claim follows immediately by combining the bounds in Theorems 4.5 and 4.8 .

Remark 4.10. Note that Theorem 4.5 follows from elementary considerations in hyperbolic geometry. In order to prove Theorem 4.8, we needed the representation of the canonical Green's function in terms of the hyperbolic Green's function, which we proved in Theorem 3.8. All quantities from hyperbolic geometry that appear in the definition for $A_{X, \varepsilon, \delta}$ are well-known invariants except for $c_{X}$. However, it has been recognized for some time that $c_{X}$ and $Z_{X}^{\prime}(1)$ are global hyperbolic invariants, which determine the complexity of the Riemann surface $X$.

\section{Uniform bounds for families of Riemann surfaces}

In this section, we study the upper bounds obtained in Theorems 4.5, 4.8, and 4.9 for certain sequences of compact Riemann surfaces. For the purpose of notational convenience, we use the following definition.

Definition 5.1. Let $\left\{X_{N}\right\}$, indexed by $N \in \mathcal{N} \subseteq \mathbb{N}$, be a sequence of compact Riemann surfaces of genus $g_{X_{N}}>1$ equipped with the hyperbolic metric $\mu_{\text {hyp }}$. We say that the sequence is admissible, if it is of one of the following two types: 


\section{J. Jorgenson And J. KRAmer}

(i) $\mathcal{N}=\mathbb{N}$, and for each $N \in \mathcal{N}$, the compact Riemann surface $X_{N+1}$ is a finite degree cover of $X_{N}$;

(ii) the sequence is the subsequence of one of the families of modular curves $\left\{X_{0}(N)\right\},\left\{X_{1}(N)\right\}$, or $\{X(N)\}$ consisting of those modular curves having genus bigger than one.

Denote by $p_{0} \in \mathcal{N}$ the minimal element in case (i), that is, $p_{0}=0$, and the smallest prime in $\mathcal{N}$ in case (ii).

Remark 5.2. In this section, we study the bounds stated in Theorems 4.5, 4.8, and 4.9 for admissible sequences of compact Riemann surfaces. The purpose is to determine the extent to which the derived bounds are uniform for all elements in the admissible sequence. We denote any bound by $O_{p_{0}}$, which signifies an implied constant being universal for all Riemann surfaces in the admissible sequence $\left\{X_{N}\right\}_{N \in \mathcal{N}}$ under consideration. Similar notation is used to denote constants, say $c\left(p_{0}\right)$, whose dependence is universal for all elements in the admissible sequence.

Lemma 5.3. Let $\left\{X_{N}\right\}_{N \in \mathcal{N}}$ be an admissible sequence of compact Riemann surfaces. Then, the hyperbolic invariants defined in $\S 2.6$ satisfy the following bounds:

(a) there is a constant $C_{1}=C_{1}\left(p_{0}\right)>0$ such that for all $N \in \mathcal{N}$, we have $\ell_{X_{N}, 0} \geqslant C_{1}$;

(b) there is a constant $C_{2}=C_{2}\left(p_{0}\right)>0$ such that for all $N \in \mathcal{N}$, we can take $r_{X_{N}}=C_{2}$;

(c) there is a constant $C_{3}=C_{3}\left(p_{0}\right)>0$ such that for all $N \in \mathcal{N}$, we have $d_{X_{N}} \leqslant C_{3}$;

(d) there is a constant $C_{4}=C_{4}\left(p_{0}\right)>0$ such that for all $N \in \mathcal{N}$, we have $C_{X_{N}}^{H K} \leqslant C_{4}$;

(e) there is a constant $C_{5}=C_{5}\left(p_{0}\right)>0$ such that for all $N \in \mathcal{N}$, we have $c_{X_{N}} \leqslant C_{5} \cdot g_{X_{N}} / \lambda_{X_{N}, 1}$.

Proof. Let us first prove the results for an admissible sequence of compact Riemann surfaces of type (i) and then consider the case of an admissible sequence of type (ii), that is, the sequences of modular curves. In order to prove the lemma for an admissible sequence of compact Riemann surfaces of type (i), we have to consider the pair of compact Riemann surfaces $X_{N}(N \in \mathbb{N})$ and $X_{0}$, where $X_{N}$ is a finite degree cover of $X_{0}$.

By taking $C_{1}=\ell_{X_{0}, 0}$, part (a) follows from the observation that $\ell_{X_{N}, 0} \geqslant \ell_{X_{0}, 0}$. Since the only requirement on $r_{X_{N}}$ is that $r_{X_{N}} \in\left(0, \ell_{X_{N}, 0}\right)$, part (b) follows from part (a) by choosing, for example, $C_{2}=C_{1} / 2$. The bound in part (c) is stated as the main theorem in [Don96] (see also [JK04a]). For part (d), we argue as follows. As usual, we have $X_{N}=\Gamma_{N} \backslash \mathbb{H}$ and $X_{0}=\Gamma_{0} \backslash \mathbb{H}$ for suitable subgroups $\Gamma_{N}$ and $\Gamma_{0}$ in $\mathrm{PSL}_{2}(\mathbb{R})$. Since $\Gamma_{N}$ is a subgroup of $\Gamma_{0}$, we have the trivial bound $K_{X_{N}}(t ; z) \leqslant K_{X_{0}}(t ; z)$, from which part (d) follows by taking $C_{4}=C_{X_{0}}^{H K}$. Finally, for part (e), we refer to the main results in [JK01], where upper and lower bounds for $c_{X_{N}}$ are proved. The upper bound stated here comes from the proof of Theorem 4.7 in [JK01]. In particular, one has to use the top displayed line on p. 21 of [JK01] with $\delta=5$ and $\varepsilon \in(0, \alpha)$, $\alpha=\min \left\{7 / 64, \lambda_{X_{N}, 1}\right\}$. From this point on, one then uses the following bounds: the number of small eigenvalues less than $\varepsilon$ is one, namely the zero eigenvalue; the number of elements in $H\left(\Gamma_{N}\right)$ of length at most five is bounded by $O_{p_{0}}\left(g_{X_{N}}\right)$, as argued in the proof of Theorem 4.11 in [JK01]; and the constant $C_{X_{N}, \varepsilon}$ defined on p. 20 in [JK01] is bounded by $O_{p_{0}}\left(g_{X_{N}}\right)$, which is proved by combining the main result in [JK02] and the well-known estimate that the number of eigenvalues less than $\frac{1}{4}$ is $O\left(g_{X_{N}}\right)$, with an implied constant that is universal. We also refer to [JK04b, Proposition 4.2], for a proof of part (e).

Let us now consider the stated assertions for the admissible sequences of modular curves. For this, complete proofs of parts (a), (c), and (e) are given in [JK04b, Proposition 5.3] for the sequence of modular curves $\left\{X_{0}(N)\right\}_{N \in \mathcal{N}}$, while part (b) again follows directly from part (a). The proof of all parts of Proposition 5.3 in [JK04b] extend with only notational changes to the other sequences of modular curves $\left\{X_{1}(N)\right\}_{N \in \mathcal{N}}$ (respectively $\{X(N)\}_{N \in \mathcal{N}}$ ); one only has to observe that, if $p$ is a 


\section{Bounds on CANONICAL GREEN's FUnCTIONS}

prime in $\mathcal{N}$, then $\operatorname{deg}\left(X_{1}\left(p_{0} p\right) / X_{1}\left(p_{0}\right)\right)=O\left(g_{X_{1}(p)}\right)$ (respectively $\left.\operatorname{deg}\left(X\left(p_{0} p\right) / X\left(p_{0}\right)\right)=O\left(g_{X(p)}\right)\right)$, with implied constants that are universal. The verification of the latter claim follows directly from known formulas (see, e.g., [Shi94]).

Finally, it remains to prove part (d) for the sequences of modular curves. We give a proof of part (d) for the sequence of modular curves $\left\{X_{0}(N)\right\}_{N \in \mathcal{N}}$. For a prime $p>p_{0}$ in $\mathcal{N}$, consider the finite-degree cover $X_{0}\left(p_{0} p\right) \longrightarrow X_{0}(p)$. Since

$$
K_{X_{0}(p)}(t ; z, w)=\sum_{\gamma \in \Gamma_{0}\left(p_{0} p\right) \backslash \Gamma_{0}(p)} K_{X_{0}\left(p_{0} p\right)}(t ; z, \gamma w)
$$

by the existence and uniqueness of heat kernels, we find

$$
K_{X_{0}(p)}(t ; z) \leqslant \frac{1}{2} \sum_{\gamma \in \Gamma_{0}\left(p_{0} p\right) \backslash \Gamma_{0}(p)}\left(K_{X_{0}\left(p_{0} p\right)}(t ; z)+K_{X_{0}\left(p_{0} p\right)}(t ; \gamma z)\right) .
$$

This shows

$$
C_{X_{0}(p)}^{H K} \leqslant\left(p_{0}+1\right) \cdot C_{X_{0}\left(p_{0} p\right)}^{H K} .
$$

Using the trivial inequality $C_{X_{0}\left(p_{0} p\right)}^{H K} \leqslant C_{X_{0}\left(p_{0}\right)}^{H K}$, we get $C_{X_{0}(p)}^{H K} \leqslant\left(p_{0}+1\right) \cdot C_{X_{0}\left(p_{0}\right)}^{H K}$ for all primes $p \in \mathcal{N}$. The claimed bound for $C_{X_{0}(N)}^{H K}$ now follows by the same principle as used in the proof of Proposition 5.3 in [JK04b]. The proof for the other sequences of modular curves $\left\{X_{1}(N)\right\}_{N \in \mathcal{N}}$ (respectively $\{X(N)\}_{N \in \mathcal{N}}$ ) is analogous.

Remark 5.4. The proofs of parts (a), (b), (c), and (d) in Lemma 5.3 are elementary and follow from standard arguments in hyperbolic geometry and analysis. Part (e) is considerably more involved. As can be seen from [JK01, JK04b], the bound stated in part (e) ultimately reduces to two bounds: the number of eigenvalues less than $\frac{1}{4}$ and the implied constant in the error term of the prime geodesic theorem. The latter constant is the focus of study in [JK02].

Theorem 5.5. Let $\left\{X_{N}\right\}_{N \in \mathcal{N}}$ be an admissible sequence of compact Riemann surfaces. For any $\delta>0, \varepsilon>0$ and $N \in \mathcal{N}$, we then have the estimate

$$
g_{\mathrm{hyp}, X_{N}}(z, w)-\sum_{\gamma \in S_{\Gamma_{N}}(\delta ; z, w)} g_{\mathbb{H}}(z, \gamma w)-\sum_{0<\lambda_{X_{N}, n}<\varepsilon} \frac{4 \pi}{\lambda_{X_{N}, n}} \varphi_{X_{N}, n}(z) \varphi_{X_{N}, n}(w)=O_{p_{0}, \varepsilon, \delta}(1) .
$$

Here, we have written $g_{\mathrm{hyp}, X_{N}}(z, w)$ instead of $g_{\mathrm{hyp}}(z, w)$ for the hyperbolic Green's function on $X_{N}=\Gamma_{N} \backslash \mathbb{H}$ in order to emphasize the dependence on $X_{N}$.

Proof. The bound follows directly by combining Theorem 4.5 with parts (b) and (d) of Lemma 5.3, as well as the definition of $\delta_{X}$ in terms of $r_{X}$, e.g., by simply taking $\delta_{X}=\max \left\{\delta_{0}, 4 r_{X}+5\right\}+1$ (see $\S 2.6)$.

TheOREm 5.6. Let $\left\{X_{N}\right\}_{N \in \mathcal{N}}$ be an admissible sequence of compact Riemann surfaces. For any $N \in \mathcal{N}$, we then have the estimate

$$
g_{\mathrm{can}, X_{N}}(z, w)-g_{\mathrm{hyp}, X_{N}}(z, w)=O_{p_{0}}\left(\frac{1}{g_{X_{N}}}\left(1+\frac{1}{\lambda_{X_{N}, 1}}\right)\right) .
$$

Here, we have written $g_{\mathrm{can}, X_{N}}(z, w)$ instead of $g_{\mathrm{can}}(z, w)$ for the canonical Green's function on $X_{N}$. Proof. Taking $\varepsilon<1$, using parts (b) and (d) of Lemma 5.3, and choosing $\delta=C_{1} / 2$ with the constant $C_{1}$ of Lemma 5.3(a), we derive from the explicit formula for $B_{X_{N}, \varepsilon, \delta}$ as stated in Theorem 4.5 that

$$
B_{X_{N}, \varepsilon, \delta}=O_{p_{0}}\left(1+\frac{1}{\varepsilon}\right) \text {. }
$$

Now we turn to the bound given in Theorem 4.8. Then, by taking $\varepsilon=\min \left\{\frac{1}{2}, \lambda_{X_{N}, 1} / 2\right\}$, and using parts (c), (e) of Lemma 5.3, the result follows. 


\section{J. Jorgenson AND J. KRAmer}

Corollary 5.7. Let $\left\{X_{N}\right\}_{N \in \mathcal{N}}$ be an admissible sequence of compact Riemann surfaces. For any $\delta>0$ and $N \in \mathcal{N}$, we then have the estimate

$$
g_{\text {can }, X_{N}}(z, w)-\sum_{\gamma \in S_{\Gamma_{N}}(\delta ; z, w)} g_{\mathbb{H}}(z, \gamma w)=O_{p_{0}, \delta}\left(1+\frac{1}{\lambda_{X_{N}, 1}}\right) ;
$$

again, we have written $g_{\mathrm{can}, X_{N}}(z, w)$ instead of $g_{\mathrm{can}}(z, w)$ for the canonical Green's function on $X_{N}=\Gamma_{N} \backslash \mathbb{H}$.

Proof. The claim follows by combining Theorem 5.5 with $\varepsilon=\min \left\{\frac{1}{2}, \lambda_{X_{N}, 1} / 2\right\}$ with Theorem 5.6 after having used the triangle inequality.

Corollary 5.8. Let $\left\{X_{N}\right\}_{N \in \mathcal{N}}$ be an admissible sequence of compact Riemann surfaces. For any $N \in \mathcal{N}$, we then have the estimate

$$
\max _{z \in X_{N}}\left|\phi_{\operatorname{Ar}}(z)\right|=O_{p_{0}}\left(1+\frac{1}{\lambda_{X_{N}, 1}}\right) ;
$$

here the $C^{\infty}$-function $\phi_{\text {Ar }}$ has been introduced in (1) in $\S 2.2$.

Proof. Using the known formula for $g_{\mathbb{H}}(z, w)$, as stated in $\S 2.3$, we can write

$$
g_{\mathrm{can}, X_{N}}(z, w)-g_{\mathbb{H}}(z, w)=g_{\mathrm{can}, X_{N}}(z, w)+\log |z-w|^{2}-\log |z-\bar{w}|^{2} .
$$

Therefore, when using the definition of the residual metrics as given in $\S 2.2$, we then have

$$
\begin{aligned}
\lim _{w \rightarrow z}\left(g_{\text {can }, X_{N}}(z, w)-g_{\mathbb{H}}(z, w)\right) & =\log \|d z\|_{\text {can,res }}^{2}-\log (2 \operatorname{Im}(z))^{2} \\
& =\log \left(\frac{\|d z\|_{\text {can,res }}^{2}}{\operatorname{Im}^{2}(z)}\right)-\log (4) \\
& =\log \left(\frac{\mu_{\text {hyp }}(z)}{\mu_{\text {Ar }}(z)}\right)-\log (4)=-\phi_{\operatorname{Ar}}(z)-\log (4) .
\end{aligned}
$$

From this, the asserted result follows directly from Corollary 5.7 by taking $\delta=C_{1} / 2$ (see Lemma 5.3(a)).

Lemma 5.9. Let $X$ be any of the modular curves $X_{0}(N), X_{1}(N)$, or $X(N)$ having genus bigger than one. Then, there is a constant $c>0$ satisfying $\lambda_{X, 1} \geqslant c$.

Proof. We recall from [Bro99, Theorem 3.1], that

$$
\liminf _{N \rightarrow \infty} \lambda_{X(N), 1} \geqslant \frac{5}{36} \text {. }
$$

Hence, there is a constant $c>0$, independent of $N$, such that $\lambda_{X(N), 1} \geqslant c$ for all $N>N_{0}$, for some $N_{0}$, thus, the claim holds for the modular curves $X(N)$ of genus bigger than one. Since $X(N)$ is a cover of $X_{0}(N)$ (respectively $X_{1}(N)$ ), the Raleigh quotient method for estimating eigenvalues, which shows that the smallest eigenvalue decreases through covers, now implies that $\lambda_{X(N), 1} \leqslant$ $\lambda_{X_{0}(N), 1}$ (respectively $\left.\lambda_{X(N), 1} \leqslant \lambda_{X_{1}(N), 1}\right)$, which completes the proof.

Corollary 5.10. Let $\left\{X_{N}\right\}_{N \in \mathcal{N}}$ be an admissible sequence of compact Riemann surfaces of type (ii), that is, of modular curves. For any $N \in \mathcal{N}$, we then have the following estimates:

$$
\max _{z, w \in X_{N}}\left|g_{\mathrm{can}, X_{N}}(z, w)-g_{\mathrm{hyp}, X_{N}}(z, w)\right|=O_{p_{0}}\left(\frac{1}{g_{X_{N}}}\right)
$$




$$
\begin{gathered}
\max _{z, w \in X_{N}}\left|g_{\mathrm{can}, X_{N}}(z, w)-\sum_{\gamma \in S_{\Gamma_{N}}(\delta ; z, w)} g_{\mathbb{H}}(z, \gamma w)\right|=O_{p_{0}, \delta}(1) \quad(\delta>0) ; \\
\max _{z \in X_{N}}\left|\phi_{\operatorname{Ar}}(z)\right|=O_{p_{0}}(1) .
\end{gathered}
$$

Proof. Combine Lemma 5.9 with the previous results, namely Theorem 5.6 for part (a), Corollary 5.7 for part (b), and Corollary 5.8 for part (c).

Remark 5.11. It is immediate from Theorem 5.6 and Corollaries 5.7 and 5.8 that Corollary 5.10 holds for any admissible sequence, which admits a universal non-zero arbitrary cover $X_{1}$ of $X_{0}$, we claim that

$$
\frac{1}{\lambda_{X_{1}, 1}}=O_{X_{0}}\left(g_{X_{1}}^{2}\right)
$$

For this, one applies [Cha84, Theorem 14, p. 112], which reduces the problem to that of bounding an isoperimetric constant associated to $X_{1}$ as a function of the degree $\operatorname{deg}\left(X_{1} / X_{0}\right)$, and the bound needed to prove this claim follows immediately from the definition of the isoperimetric constant in question (see also [Cha84, Theorem 12, p. 111 and Definition 5, p. 110]).

Remark 5.12. As stated in the introduction, this paper was motivated by a question from Edixhoven who asked for bounds for the canonical Green's function on $X_{1}(N)$. Recall that, as stated in the proof of Lemma 4.4, the hyperbolic Green's function $g_{\mathbb{H}}(z, w)(z, w \in \mathbb{H})$ is expressible in terms of elementary functions. Combining this expression with Corollary 5.10(b) provides the upper and lower bounds sought by Edixhoven.

Remark 5.13. In a slightly more general situation, one can restrict attention to arbitrary compact subsets of $X_{N}$, and consider admissible sequences of non-compact hyperbolic surfaces. Beginning with Lemma 4.2 , the constant $r_{X_{N}}$ would then be bounded away from zero with a lower bound that depends on the subset of $X_{N}$ under consideration. The resulting bound for hyperbolic heat kernels and hyperbolic Green's functions then can be applied throughout the subsequent calculations. By doing so, one can address the problem of understanding the asymptotic behavior of the canonical Green's function for a degenerating family of algebraic curves approaching the Deligne-Mumford boundary of the moduli space of stable curves of a fixed positive genus, as first studied in [Jor90].

Remark 5.14. In his recent work [Küh05], Kühn used the analysis of the present paper and from [JK04a] to derive bounds for the arithmetic self-intersection number of the relative dualizing sheaf on an arithmetic surface. By revisiting the analytic component of the computations in [AU97], he is able to both simplify the method of proof given in [AU97] and to provide a technique which extends to the modular curves $X_{1}(N)$ and $X(N)$.

\section{ACKNOWLEDGEMENTS}

We thank B. Edixhoven for discussing the applications of the present article to his own work and, specifically, for providing us with a written description of his ideas. This description formed the basis of $\S 1.2$. At one time, B. Edixhoven posed the question of deriving upper bounds for the canonical Green's functions on the modular curves $\left\{X_{1}(N)\right\}$ to P. Sarnak, who forwarded Edixhoven's question to us. We thank P. Sarnak for communicating Edixhoven's problem to us.

\section{REFERENCES}

AU97 A. Abbes and E. Ullmo, Auto-intersection du dualisant relatif des courbes modulaires $X_{0}(N)$, J. reine angew. Math. 484 (1997), 1-70. 


\section{Bounds on CANONICAL GREEN'S FUnCTIONS}

Ara74 S. Arakelov, Intersection theory of divisors on an arithmetic surface, Math. USSR Izv. 8 (1974), 1167-1180.

Bea95 A. Beardon, The geometry of discrete groups, Graduate Texts in Mathematics, vol. 91 (Springer, Berlin, 1995).

Bro99 R. Brooks, Platonic surfaces, Comment. Math. Helv. 74 (1999), 156-170.

Cha84 I. Chavel, Eigenvalues in Riemannian geometry (Academic Press, Orlando, FL, 1984).

Don96 H. Donnelly, Elliptic operators and covers of Riemannian manifolds, Math. Z. 223 (1996), 303-308.

Hej83 D. Hejhal, The Selberg trace formula for $\mathrm{PSL}_{2}(\mathbb{R})$, vol. 2, Lecture Notes in Mathematics, vol. 1001 (Springer, Berlin, 1983).

Jor90 J. Jorgenson, Asymptotic behavior of Faltings's delta function, Duke Math. J. 61 (1990), 221-254.

JK01 J. Jorgenson and J. Kramer, Bounds on special values of Selberg's zeta functions for Riemann surfaces, J. reine angew. Math. 541 (2001), 1-28.

JK02 J. Jorgenson and J. Kramer, On the error term of the prime geodesic theorem, Forum Math. 14 (2002), 901-913.

JK04a J. Jorgenson and J. Kramer, Bounding the sup-norm of automorphic forms, Geom. Funct. Anal. 14 (2004), 1267-1277.

JK04b J. Jorgenson and J. Kramer, Bounds on Faltings's delta function through covers, Preprint (2004).

JL95 J. Jorgenson and R. Lundelius, Convergence theorems for relative spectral functions on hyperbolic Riemann surfaces of finite volume, Duke Math. J. 80 (1995), 785-819.

Küh05 U. Kühn, On an upper bound for the arithmetic self-intersection number of the dualizing sheaf on arithmetic surfaces, Preprint (2005).

Shi94 G. Shimura, Introduction to the arithmetic theory of automorphic functions (Princeton University Press, Princeton, NJ, 1994).

\section{J. Jorgenson jjorgenson@mindspring.com}

Department of Mathematics, The City College of New York, Convent Avenue at 138th Street, New York, NY 10031, USA

J. Kramer kramer@math.hu-berlin.de Institut für Mathematik, Humboldt-Universität zu Berlin, Unter den Linden 6, D-10099 Berlin, Germany 